\title{
Nota Técnica: \\ La nueva norma europea de especificaciones de cementos comunes UNE-EN 197-1:2011
}

\author{
Technical Note: \\ The new european standard on common cements specifications \\ EN 197-1:2011
}

\author{
M. A. Sanjuán(*), C. Argiz(**)
}

Recepción/Received: 27-XII-11

Aceptación/Accepted: 23-I-12

RESUMEN

En este artículo se presentan las novedades de la nueva norma europea de especificaciones de cementos comunes UNE-EN 197-1:2011 aprobada el 6 de agosto de 2011 como EN 197-1:2011 y publicada el 19 de junio de 2012 en el Diario Oficial de la Unión Europea (DOUE). Esta norma se publicará en el Boletín Oficial del Estado (BOE) antes de final de año. La fecha de disponibilidad (date of applicability, DAV) es el 1 de julio de 2012 y el periodo de coexistencia finalizará el 1 de julio de 2013.

La UNE-EN 197-1:2000 fue la primera norma armonizada dentro del campo de la Directiva de Productos de la Construcción (DPC) europea y esta primera revisión incorpora unos nuevos requisitos para establecer cuándo un cemento común, además, es resistente a la acción de los sulfatos. De esta forma, se completa el mandato dado por la Comisión Europea a CEN para la elaboración de normas armonizadas de cementos (mandato M114).

Palabras clave: EN 197-1, cementos comunes, cemento resistente a los sulfatos, normalización.
SUMMARY

In this paper, the novelties of European standard EN 197-1:2011 which has been aprobed on $6^{\text {th }}$ August, 2011 are presented. The european standard EN 197-1:2011 has been published in the Official Journal of the European Union on 19th June, 2012 (C 176/1). The UNEEN 197-1:2011 will be published in Spain in the Official Journal of Spain (B.O.E.). The date of applicability (DAV) of the standard as a harmonised European standard is on $1^{\text {st }}$ of July, 2012 and the date of the end of the coexistence period is on $1^{\text {st }}$ of July, 2013.

The former EN 197-1:2000 has been the first European standard in the field of the Construction Products Directive (CPD) and this first revision include the requirements needed for a common cement to be, in addition, sulphate resisting cement. Therefore, the mandate given by the European Commission to CEN to prepare harmonised standards in the cement field (mandate M114) has been fulfilled.

Keywords: EN 197-1, common cement, sulphate resisting cement, standarization.

\footnotetext{
(*) Instituto Español del Cemento y sus Aplicaciones (IECA) (Madrid, España).

(**) Universidad Politécnica de Madrid (UPM) (Madrid, España).
} 


\section{INTRODUCCIÓN}

En agosto de 2011 se ha aprobado la primera revisión de la norma europea de especificaciones de cementos comunes UNE-EN 197-1:2000 (1, 2), la cual se denomina EN 197-1:2011 (3). Diez años después de la aparición de la UNE-EN 197-1:2000, que fue la primera norma de especificaciones armonizada de un producto de construcción dentro del campo de aplicación de la Directiva de Productos de Construcción (DPC), se ha aprobado su primera renovación. La traducción española de la EN 197-1:2011 es la UNE-EN 197-1:2011, que anula a la UNE-EN 197$1: 2000$ y a su erratum (UNE-EN 197-1:2000 erratum) $(4,5)$. La EN 197-1:2011 se ha publicado el 19 de junio de 2012 en el Diario Oficial de la Unión Europea y se publicará posteriormente en el BOE. La fecha de disponibilidad (date of applicability, DAV) es el 1 de julio de 2012 y el periodo de coexistencia finalizará el 1 de julio de 2013.

El proceso de revisión final de la EN 197-1:2000 se inició en la reunión del Plenario del CEN/TC 51 de Montreaux de 2009 con la activación del Work item 00051077 de revisión de la EN 197-1 (Resolution 513) y el envío a encuesta de la prEN 197-1 (Resolution 514). Por otro lado, el 19 de marzo de 2010 se reunió el grupo de trabajo ${ }^{1}$ CEN/TC 51/WG 6 "Definiciones y terminología del cemento", en la sede de DIN (Deutsches Institut für Normung e.V.) en Berlín, para estudiar los 196 comentarios, técnicos y editoriales resultantes de la encuesta de la prEN197-1 finalizada el 20 de enero de 2010. Veintiséis países la aceptaron como posible norma europea, tres se abstuvieron (Chipre, Irlanda y Eslovaquia) y solo Bélgica votó en contra debido a su oposición a la coexistencia de tres niveles de cementos resistentes a los sulfatos del tipo I (SR 0, SR 3 y SR 5); solo quieren un cemento CEM I-SR con un contenido de $\mathrm{C}_{3} \mathrm{~A}$ inferior o igual al $3,0 \%$.

\section{LAS NOVEDADES DE LA EN 197-1:2011}

La figura 1 presenta un esquema que muestra la composición de la UNE-EN 197-1:2011 en la que se incorporan las normas UNE-EN 197-1:2000/A1:2004 (Cemento LH),
UNE-EN 197-1:2000/A3:2007 (Cenizas volantes silíceas, V) y UNE-EN 197-4:2004 (Cementos de escorias de horno alto de baja resistencia inicial, LES) junto con los borradores de norma EN 197-1/prA2 (Cementos comunes resistentes a los sulfatos) y EN 197-4/prA1 (Cementos de escorias de horno alto de baja resistencia inicial resistentes a los sulfatos) (6-11).

La primera novedad del capítulo 5 "componentes" está en los límites de composición del CEM II/A-M que se explican mejor que en la UNE-EN 197-1:2000 y se detalla que el contenido de clínker está establecido en el rango de 80 a 88 , en vez de $80-94$, quedando el límite de adiciones limitado del 12 al 20, en vez de 6-20, es decir:

EN 197-1:2000 $\rightarrow$ CEM II/A-M: $K=80-94$ y $M=6-20$

EN 197-1:2011 $\rightarrow$ CEM II/A-M: $K=80-88$ y $M=12-20$

También se incorpora un nuevo requisito que deben cumplir los clínkeres con los que se van a fabricar los cementos resistentes a los sulfatos, se trata del límite superior del contenido de $\mathrm{C}_{3} \mathrm{~A}$ que se define en un nuevo capítulo 7.4 "Requisitos de durabilidad". Los cementos Portland comunes resistentes a los sulfatos y los cementos puzolánicos resistentes a los sulfatos se elaboran con clínker de cemento Portland en el cual el contenido de $\mathrm{C}_{3} \mathrm{~A}$ no excede:

- Para el CEM I: 0\%, 3\% o 5\%, según corresponda.

- Para el CEM IV/A y CEM IV/B: $9 \%$.

El contenido de aluminato tricálcico $\left(\mathrm{C}_{3} \mathrm{~A}\right)$ en el clínker conforme con la norma europea se calculará con la ecuación [1]:

$$
C_{3} A=2,65 \cdot A-1,69 \cdot F(\%)
$$

En donde $\mathrm{A}$ es el porcentaje en masa del óxido de aluminio $\left(\mathrm{Al}_{2} \mathrm{O}_{3}\right)$ del clínker y $\mathrm{F}$ es el porcentaje en masa del óxido de hierro (III) $\left(\mathrm{Fe}_{2} \mathrm{O}_{3}\right)$ del clínker, ambos determinados de acuerdo con la EN 196-2. Puede suceder que se obtenga un valor negativo de $\mathrm{C}_{3} \mathrm{~A}$ en el cálculo, en este caso, se registrará el valor del $0 \%$.

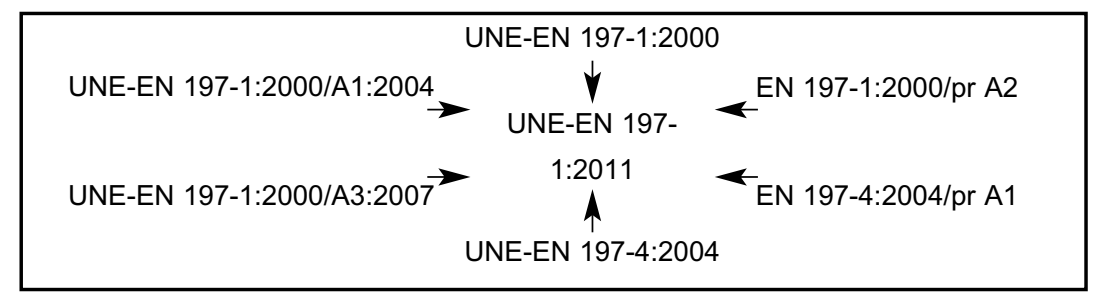

Figura 1. Composición de la UNE-EN 197-1:2011.

1 Los representantes españoles en el grupo de trabajo del Comité Europeo de Normalización CEN/TC 51/WG 6 "Definiciones y terminología del cemento" son D. Juan Puig Montraveta, Presidente del Subcomité no 3 del AEN/CTN-80: "Definiciones, terminología y especificaciones" desde el 11 de abril de 2007, y Miguel Ángel Sanjuán Barbudo, Secretario del mismo Subcomité nº 3 del AEN/CTN-80 desde el 22 de octubre de 2004. 
En la norma española UNE 80304:2006 (12) el contenido de aluminato tricálcico $\left(C_{3} A\right)$ en el clínker se calcula con la misma fórmula de la norma europea y el contenido de ferrito-aluminato tetracálcico $\left(\mathrm{C}_{4} \mathrm{AF}\right)$ en el clínker se calcula con la fórmula siguiente [2]:

$$
\mathrm{C}_{4} \mathrm{AF}=3,04 \cdot \mathrm{F}(\%)
$$

Otra novedad importante es que, en el caso particular del CEM I, es permisible el cálculo del contenido de $\mathrm{C}_{3} \mathrm{~A}$ del clínker a partir del análisis químico del cemento. Actualmente, el CEN/TC 51 está desarrollando un método de ensayo para determinar el contenido de $C_{3} A$ del clínker a partir del análisis de muestras puntuales de cemento. Sin embargo, hasta que este método esté disponible, el contenido de $\mathrm{C}_{3} \mathrm{~A}$ debería determinarse directamente en el clínker. La frecuencia mínima de ensayo y la utilización de métodos alternativos para la evaluación directa o indirecta del $\mathrm{C}_{3} \mathrm{~A}$ debería de incluirse en el control de producción de fábrica, aunque una frecuencia de ensayo habitual es de dos al mes.

Por otro lado, el límite de la adición principal de los cementos comunes CEM IV/A comprende el rango del $11 \%$ al $35 \%$. Para los cementos comunes resistentes a los sulfatos CEM IV/A-SR se propuso elevar el límite inferior. Finalmente, a propuesta de España, este límite no se ha elevado todo lo que en un principio se propuso y, en consecuencia, el rango ha quedado establecido del $21 \%$ al $35 \%$.

Una novedad importante en el capítulo 5 de "componentes" de los cementos es la reducción del límite superior de los aditivos orgánicos que pasa de $0,5 \%$ en la EN 197-1:2000 a 0,2\% en la UNE-EN 197-1:2011. Por otro lado, el límite superior de la cantidad total de aditivos se mantiene en $1,0 \%$.

Cuando en la misma fábrica, un fabricante produce diferentes cementos que corresponden a la misma designación normalizada, estos cementos reciben una identificación adicional en forma de un número o de dos letras minúsculas, entre paréntesis, con el fin de distinguir estos cementos unos de otros. Para el sistema numérico, este número debería ser el 1 para el segundo cemento certificado, 2 para el siguiente y así sucesivamente. Para el sistema de letras, estas se deben elegir de tal forma que se evite cualquier confusión.

\section{INCORPORACIÓN DE LOS CEMENTOS COMUNES RESISTENTES A LOS SULFATOS (SRC)}

Los requisitos relativos a la influencia del cemento en la durabilidad de los productos construidos con él se han dirigido a las normas y reglamentos del hormigón aplicables en el lugar de uso tanto en la norma UNE-EN 197-1:2000 como en la UNE-EN 197-1:2011. Sin embargo, en cuanto a las propiedades durables hay una novedad muy importante en la UNE-EN 197-1:2011 ya que incorpora unos nuevos requisitos para establecer cuándo un cemento común, además, es resistente a la acción de los sulfatos. En consecuencia, la novedad reside en la inclusión de un apartado específico dedicado a los cementos resistentes a los sulfatos cuya utilización se recomienda en los hormigones expuestos a los ambientes que contengan una elevada concentración de iones sulfato como sucede en los ambientes marinos y cuando los hormigones se encuentran en contacto con terrenos yesíferos. Asimismo, la designación normalizada de los cementos comunes resistentes a los sulfatos es otra novedad.

La futura posible predicción de las propiedades mecánicas del cemento a nivel microstructural (13), posiblemente propicie la aparición de nuevas normas que consideren características microestructurales que sean medidas con técnicas de medida diferentes a las que se emplean ahora en el campo de la normalización, como por ejemplo, técnicas de difracción de rayos X (14), métodos electromagnéticos (15), etc.

En el capítulo 6 de composición y de denominación de los cementos hay una novedad muy importante ya que se incorporan a esta norma los cementos resistentes a los sulfatos (Tabla 1) considerando que un cemento común es resistente a los sulfatos cuando cumple con una exigencia relativa a un límite superior en el contenido de $\mathrm{C}_{3} \mathrm{~A}$ calculado como $\mathrm{C}_{3} \mathrm{~A}=2,65 \mathrm{Al}_{2} \mathrm{O}_{3}-1,69 \mathrm{Fe}_{2} \mathrm{O}_{3}$ y definido en la Tabla 1.

Tabla 1. Limitación de $C_{3} A$ en el clínker en los cementos comunes resistentes a los sulfatos (SRC).

\begin{tabular}{|c|c|}
\hline $\begin{array}{c}\text { Cementos comunes resistentes } \\
\text { a los sulfatos }\end{array}$ & $\begin{array}{c}\mathbf{C}_{3} \mathbf{A}(\%) \\
\text { en el clínker }\end{array}$ \\
\hline CEM I-SR 0 & $=0 \%$ \\
\hline CEM I-SR 3 & $\leq 3 \%$ \\
\hline CEM I-SR 5 & $\leq 5 \%$ \\
\hline CEM III/B-SR & Sin requisito \\
\hline CEM III/C-SR & Sin requisito \\
\hline CEM IV/A-SR & $\leq 9 \%$ \\
\hline CEM IV/B-SR & $\leq 9 \%$ \\
\hline
\end{tabular}

Hay que destacar las peculiaridades de la composición de los cementos resistentes a los sulfatos de la Tabla 2 en comparación con la composición de los cementos comunes como ya se ha comentado anteriormente:

Los cementos CEM IV/A-SR deben contener del $65 \%$ al $79 \%$ de clínker, por lo que el límite superior es diez puntos porcentuales menor que el clínker $(K=65-89 \%)$ de los CEM IV/A que no son resistentes a los sulfatos.

Las únicas adiciones permitidas son las escorias de horno alto, las puzolanas naturales y las cenizas volantes silíceas. 
Tabla 2. Los siete productos de la familia de los cementos comunes resistentes a los sulfatos.

\begin{tabular}{|c|c|c|c|c|c|c|c|}
\hline \multirow{3}{*}{$\begin{array}{l}\text { Tipos } \\
\text { principales }\end{array}$} & \multirow{3}{*}{\multicolumn{2}{|c|}{$\begin{array}{l}\text { Designación de los } 7 \text { productos } \\
\text { (tipos de cementos comunes) }\end{array}$}} & \multicolumn{5}{|c|}{ Composición (proporción en masa¹)) } \\
\hline & & & \multicolumn{4}{|c|}{ Componentes principales } & \multirow[b]{2}{*}{$\begin{array}{l}\text { Constit. } \\
\text { Minorit. }\end{array}$} \\
\hline & & & Clínker K & $\begin{array}{l}\text { Escorias de } \\
\text { Horno Alto S }\end{array}$ & Puzolana & $\begin{array}{l}\text { Cenizas } \\
\text { Volantes }\end{array}$ & \\
\hline CEM I & $\begin{array}{l}\text { Cemento Portland } \\
\text { resistente a los sulfatos }\end{array}$ & $\begin{array}{l}\text { CEM I-SR } 0 \\
\text { CEM I-SR } 3 \\
\text { CEM I-SR } 5\end{array}$ & $95-100$ & & & & $0-5$ \\
\hline \multirow{2}{*}{ CEM III } & \multirow{2}{*}{$\begin{array}{l}\text { Cemento de horno alto } \\
\text { resistente a los sulfatos }\end{array}$} & CEM III/B-SR & $20-34$ & $66-80$ & - & - & $0-5$ \\
\hline & & CEM III/C-SR & 5-19 & $81-95$ & - & - & $0-5$ \\
\hline \multirow{2}{*}{ CEM IV } & \multirow{2}{*}{$\begin{array}{l}\text { Cemento puzolánico } \\
\text { resistente a los sulfatos²) }\end{array}$} & CEM IV/A-SR & $65-79$ & & \multicolumn{2}{|c|}{$21-35$} & $0-5$ \\
\hline & & CEM IV/B-SR & $45-64$ & & \multicolumn{2}{|c|}{$36-55$} & $0-5$ \\
\hline
\end{tabular}

1) Los valores de la tabla se refieren a la suma de los componentes principales y minoritarios.

2) En los cementos puzolánicos resistentes a los sulfatos, los tipos CEM IV/A-SR y CEM IV/B-SR, los componentes principales además del clínker deben ser declarados en la denominación del cemento (véase apartado 8 de la EN 197-1).

Por otro lado, el CEN/TC 51 ha decidido la implantación de un sistema de seguimiento de la implementación de la UNE-EN 197-1:2011 con relación a los cementos resistentes a los sulfatos en los Estados miembros, trabajo que se ha encargado a CEMBUREAU ya que dispone de las estadísticas europeas de producción y consumo de cementos. Asimismo, se permite la coexistencia de cementos resistentes a los sulfatos recogidos en las normas nacionales de los Estados miembros y recogidas en el anejo A de la UNE-EN 197-1:2011. En particular, en España se incluirán en la próxima revisión de la UNE 80303-1 $(16,17)$, los cementos de la Tabla 3.

Tabla 3. Cementos españoles del anejo A de la UNE-EN 197-1:2011.

\begin{tabular}{|c|c|c|}
\hline $\begin{array}{c}\text { Países miembros } \\
\text { del CEN }\end{array}$ & Normas nacionales & $\begin{array}{c}\text { Tipos de cemento } \\
\text { CEM }\end{array}$ \\
\hline \multirow[t]{3}{*}{ España } & \multirow[t]{3}{*}{ UNE $80303-1$} & $\begin{array}{c}\text { II/A-S; II/B-S, II/A-D, } \\
\text { II/A-P, II/B-P, II/A-V, } \\
\text { II/B-V }\end{array}$ \\
\hline & & III/A \\
\hline & & $\mathrm{V} / \mathrm{A}$ \\
\hline
\end{tabular}

\subsection{Especificaciones químicas de los cementos comunes resistentes a los sulfatos}

A los cementos resistentes a los sulfatos se les exige un nuevo requisito (límite superior en el contenido de $C_{3} A$ ), como ya se ha adelantado en los apartados anteriores, y dos requisitos que también se piden al resto de los cementos comunes (contenido de sulfatos $-\mathrm{SO}_{3}-$ y puzolanicidad -solo en los CEM IV-) pero con un nivel de exigencia superior; es decir, el contenido de sulfatos debe ser medio punto porcentual inferior que para el resto de los cementos comunes y el ensayo de puzolanicidad, en el caso de los cementos CEM IV/A-SR y CEM IV/B-SR debe ser positivo a los 8 días. La Tabla 4 recoge las exigencias adicionales de los cementos comunes resistentes a los sulfatos que se resumen en la Figura 2:

- Nuevo requisito del contenido de $\mathrm{C}_{3} \mathrm{~A}$ (límite superior).

- Requisito más exigente del contenido de $\mathrm{SO}_{3}$ (límite superior).

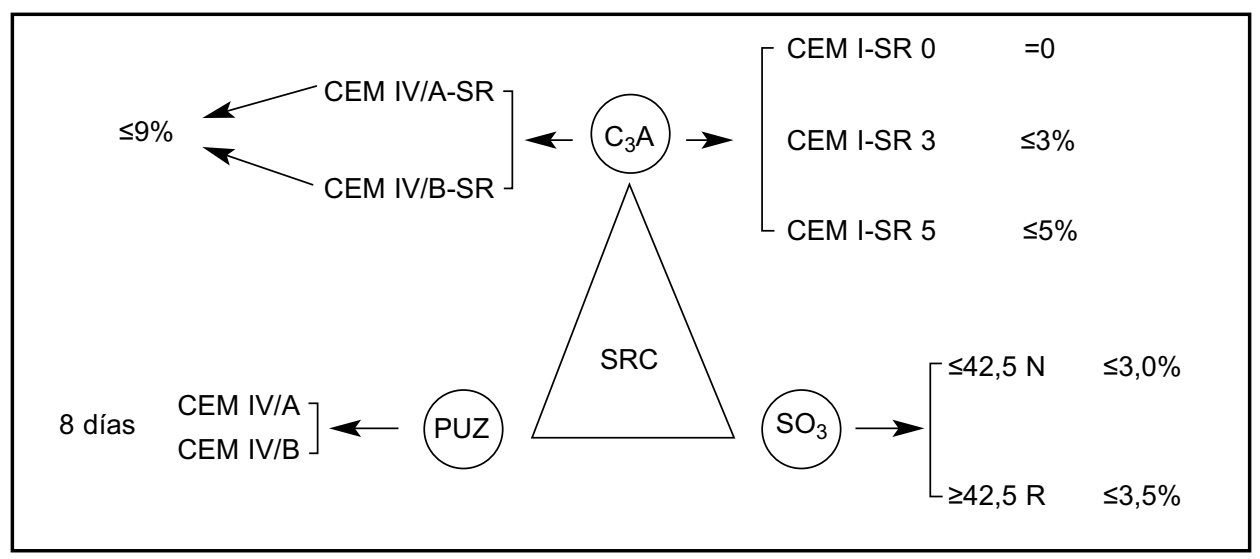

Figura 2. Exigencias adicionales de los cementos comunes resistentes a los sulfatos. 
- Requisito más exigente de la puzolanicidad en los CEM IV/A-SR y CEM IV/B-SR: cumplimiento del ensayo a 8 días.

Finalmente, conviene remarcar de nuevo que el límite inferior del contenido de $\mathrm{P}+\mathrm{V}$ de los cementos comunes puzolánicos CEM IV/A-SR resistentes a los sulfatos es de $21 \%$ en vez del $11 \%$ especificado por el resto de los cementos puzolánicos del tipo CEM IV/A.

\section{PLAZOS}

De acuerdo con el Comité Europeo de Normalización (CEN), la norma europea de especificaciones de cementos comunes EN 197-1:2011 se ha traducido al español antes del 31 de marzo de 2012 y su implementación a nivel nacional deberá realizarse antes del 30 de junio de 2013. Asimismo, la revisión de la norma europea de especificaciones de cementos comunes UNE-EN 1971:2011 se deberá de iniciar el 14 de septiembre de 2016.

AENOR ha traducido la norma europea de especificaciones de cementos comunes EN 197-1:2011 en plazo (28 de diciembre de 2011) y la ha publicado como UNE-EN 197-1:2011. Finalmente, la EN 197-1:2011 se ha publicado el 19 de junio de 2012 en el Diario Oficial de la Unión Europea y, posteriormente, se publicará en el BOE.

\section{CONCLUSIÓN}

La EN 197-1:2011 se ha publicado el 19 de junio de 2012 en el Diario Oficial de la Unión Europea y se publicará en el $\mathrm{BOE}$, posiblemente, un par de meses después. La fecha de disponibilidad (date of applicability, DAV) es el 1 de julio de 2012 y el periodo de coexistencia finalizará el 1 de julio de 2013.

La norma europea UNE-EN 197-1:2011 completa el trabajo de normalización de los cementos comunes resistentes a los sulfatos de acuerdo con el Mandato M/114 de la Comisión Europea al Comité Europeo de Normalización (CEN).

En el futuro de la norma europea de especificaciones de cementos comunes UNE-EN 197-1 se debe de fomentar la normalización de nuevas adiciones, especialmente las procedentes de residuos industriales, que se puedan considerar homogéneas en el tiempo y no perjudiciales, por un lado, y la optimización del empleo de las adiciones ya normalizadas, por otro.

\section{AGRADECIMIENTOS}

Los autores agradecen a Olga Fernández Hernández su colaboración en la edición del presente artículo.

Tabla 4. Requisitos adicionales a los cementos comunes resistentes a los sulfatos dados como valores característicos.

\begin{tabular}{|c|c|c|c|c|}
\hline Propiedades & Ensayo de referencia & Tipo de cemento & Clase de resistencia & Requisitos $^{\text {a) }}$ \\
\hline \multirow{2}{*}{$\begin{array}{l}\text { Contenido de sulfatos } \\
\text { (expresado como } \mathrm{SO}_{3} \text { ) }\end{array}$} & \multirow{2}{*}{ EN $196-2$} & \multirow{2}{*}{$\begin{array}{l}\text { CEM I-SR } 0 \\
\text { CEM I-SR } 3 \\
\text { CEM I-SR 5b } \\
\text { CEM IV/A-SR } \\
\text { CEM IV/B-SR }\end{array}$} & $\begin{array}{l}32,5 \mathrm{~N} \\
32,5 \mathrm{R} \\
42,5 \mathrm{~N}\end{array}$ & $\leq 3.0 \%$ \\
\hline & & & $\begin{array}{l}42,5 \mathrm{R} \\
52,5 \mathrm{~N} \\
52,5 \mathrm{R}\end{array}$ & $\leq 3.5 \%$ \\
\hline \multirow{4}{*}{$\mathrm{C}_{3} \mathrm{~A}$ en el Clínker } & \multirow{4}{*}{ EN 196-2 } & CEM I-SR 0 & \multirow{4}{*}{ Todas } & $=0^{d}$ \\
\hline & & CEM I-SR 3 & & $\leq 3 \%$ d \\
\hline & & CEM I-SR 5 & & $\leq 5 \%{ }^{d}$ \\
\hline & & $\begin{array}{l}\text { CEM IV/A-SR } \\
\text { CEM IV/B-SR }\end{array}$ & & $\leq 9 \% d$ \\
\hline Puzolanicidad & EN $196-5$ & $\begin{array}{l}\text { CEM IV/A-SR } \\
\text { CEM IV/B-SR }\end{array}$ & Todas & $\begin{array}{c}\text { Cumplir con el Ensayo } \\
\text { a los } 8 \text { días }\end{array}$ \\
\hline
\end{tabular}

a) Las exigencias se dan en porcentaje en masa del cemento final o clínker tal y como se define en la tabla.

b) Se puede producir un CEM I-SR 5 con un contenido de sulfatos superior para algunas aplicaciones específicas. En tal caso, se debe declarar en el albarán de entrega el valor numérico superior del contenido de sulfatos.

c) El contenido de aluminato tricálcico se debe de calcular con la fórmula: $C_{3} A=2,65$ A-1,69F (véase 5.2.1 de la EN 197-1).

d) El método de ensayo para la determinación del contenido de $C_{3} A$ en el clínker mediante el análisis del cemento final está realizándose en el CEN/TC $51 /$ WG 15 .

\section{BIBLIOGRAFÍA}

(1) Sanjuán, M. A. y Calleja, J.: "De las normas de especificaciones de cementos de 1996 a las correspondientes de 2000 y 2001". Cemento-Hormigón, no 836, mayo 2002, pp. 20-27.

(2) Sanjuán, M. A.: Revisión de la norma europea de especificaciones de cementos comunes EN 197-1:2000. Cemento-hormigón, no 941, noviembre-diciembre 2010. ISSN: 0008-8919, pp. 18-33. 
(3) EN 197-1:2011 - Cement - Part 1: Composition, specifications and conformity criteria for common cement (aprobada el 6 de agosto de 2011).

(4) UNE-EN 197-1:2000 - Cemento. Parte 1: Composición, especificaciones y criterios de conformidad de los cementos comunes, 29 pp.

(5) UNE-EN 197-1:2000 erratum - Cemento. Parte 1: Composición, especificaciones y criterios de conformidad de los cementos comunes, $7 \mathrm{pp}$.

(6) UNE-EN 197-1:2000/A1:2005 - Cemento. Parte 1: Composición, especificaciones y criterios de conformidad de los cementos comunes. (Cementos LH), $12 \mathrm{pp}$.

(7) EN 197-1:2000/prA2:2010 - Cemento. Parte 1: Composición, especificaciones y criterios de conformidad de los cementos comunes (Cementos SRC).

(8) UNE-EN 197-1:2000/A3:2008 - Cemento. Parte 1: Composición, especificaciones y criterios de conformidad de los cementos comunes (Cenizas V), $7 \mathrm{pp}$.

(9) UNE-EN 197-4:2005 - Cemento. Parte 4: Composición, especificaciones y criterios de conformidad de cementos de horno alto de baja resistencia inicial, $24 \mathrm{pp}$.

(10) EN 197-4:2005/prA1:2010 - Cemento. Parte 4: Composición, especificaciones y criterios de conformidad de cementos de horno alto de baja resistencia inicial (SRC).

(11) UNE-EN 14216:2005 - Cemento. Composición, especificaciones y criterios de conformidad de cementos especiales con bajo calor de hidratación.

(12) UNE 80304:2006 - Cementos. Cálculo de la composición potencial del clínker portland.

(13) Qian, Z.; Schlangen, E.; Ye, G.; Breugel, K. van.: "Prediction of mechanical properties of cement paste at microscale", Mater. Construcc., vol. 60, 297, 7-18. enero-marzo 2010.

(14) Manzano, H.; González-Teresa, R.; Dolado, J. S.; Ayuela, A.: "X-ray spectra and theoretical elastic properties of crystalline calcium silicate hydrates: comparison with cement hydrated gels", Mater. Construcc., vol. 60, 299, 7-19, julio-septiembre 2010.

(15) Zornoza, E.; Catalá, G.; Jiménez, F.; Andión, L. Ga; Garcés, P.: "Electromagnetic interference shielding with Portland cement paste containing carbon materials and processed fly ash", Mater. Construcc., vol. 60, 300, 21-32. octubre-diciembre 2010.

(16) UNE 80303-1:2001 - Cementos con características adicionales. Parte 1: Cementos resistentes a los sulfatos.

(17) UNE 80303-1:2001/1M:2006 - Cementos con características adicionales. Parte 1: Cementos resistentes a los sulfatos $1^{\text {a }}$ Modificación. 